\title{
Comparison of Regional Brain Volume and Glucose Metabolism Between Patients with Mild Dementia with Lewy Bodies and Those with Mild Alzheimer's Disease
}

\author{
Kazunari Ishii ${ }^{1}$, Tsutomu Soma ${ }^{2,3}$, Atsushi K. Kono ${ }^{1}$, Keitaro Sofue ${ }^{1}$, Naokazu Miyamoto ${ }^{1}$, Toshiki Yoshikawa ${ }^{1}$, \\ Etsuro Mori ${ }^{4}$, and Kenya Murase ${ }^{5}$ \\ ${ }^{I}$ Department of Radiology and Nuclear Medicine, Hyogo Brain and Heart Center, Himeji, Hyogo, Japan; ${ }^{2}$ Department of \\ Medical Engineering, School of Allied Health Sciences, Faculty of Medicine, Osaka University, Suita, Osaka, Japan; ${ }^{3}$ Clinical \\ Application Technology Group, Daiichi Radioisotope Laboratories, Tokyo, Japan; ${ }^{4}$ Behavioral Neurology and Cognitive \\ Neuroscience, Tohoku University Graduate School of Medicine, Sendai, Miyagi, Japan; and ${ }^{5}$ Division of Medical Technology \\ and Science, Health Science Course, Department of Medical Physics and Engineering, Graduate School of Medicine, \\ Osaka University, Suita, Osaka, Japan
}

The aim of this study was to investigate regional differences between morphologic and functional changes in patients with mild dementia with Lewy bodies (DLB) compared with those with Alzheimer's disease (AD). Methods: Twenty patients with very mild DLB (mean age, 74.5 y; mean Mini-Mental State Examination [MMSE] score, 24.0), 20 patients with very mild AD (mean age, $74.1 \mathrm{y}$; mean MMSE score, 24.0), and 20 age- and sex-matched healthy volunteers (normal controls [NC]) underwent both ${ }^{18} \mathrm{~F}-\mathrm{FDG}$ PET and 3-dimensional spoiled gradient echo MRI. Fully automatic volumetry of the MRI data was used to obtain whole brain, hippocampal, occipital, and striatal volumes, which were compared with the results of a similar analysis of glucose metabolic data. Results: In DLB patients, volumetric data indicated a significant volume decrease in the striatum, whereas ${ }^{18} \mathrm{~F}-\mathrm{FDG}$ PET showed significant glucose metabolic reductions in the temporal, parietal, and frontal areas _ including in the occipital lobe - compared with those in the NC group. In contrast, in AD patients, both the hippocampal volume and glucose metabolism were significantly decreased, whereas the occipital volume and metabolism were preserved. Conclusion: Comparison of very mild DLB and AD revealed different morphologic and metabolic changes occurring in the medial temporal lobes and the occipital lobe, demonstrating characteristic pathophysiologic differences between these 2 diseases.

Key Words: dementia; Lewy bodies; Alzheimer's disease; ${ }^{18} \mathrm{~F}-$ FDG; PET; MRI; glucose metabolism; volumetry

J Nucl Med 2007; 48:704-711

DOI: 10.2967/jnumed.106.035691

Received Aug. 17, 2006; revision accepted Jan. 29, 2007

For correspondence or reprints contact: Kazunari Ishii, MD, Department of Radiology and Nuclear Medicine, Hyogo Brain and Heart Center, 520 SaishoKo, Himeji, Hyogo 670-0981, Japan.

E-mail: kishii@hbhc.jp

COPYRIGHT @ 2007 by the Society of Nuclear Medicine, Inc.
D ementia with Lewy bodies (DLB), a clinical entity of primary degenerative dementia pathologically characterized by the presence of Lewy bodies in cortical, subcortical, and brain-stem structures, is the second most common degenerative dementia after Alzheimer's disease (AD). In the DLB brain, the occipital lobe is not affected histopathologically but metabolic or perfusional reduction is present in the occipital lobe (1-3), whereas the medial temporal limbic area is minimally affected histopathologically (4-6). In the mild $\mathrm{AD}$ brain, neuropathologic changes predominantly affect the neocortical association area and medial temporal limbic area. These histopathologic changes lead to focal atrophy and can be demonstrated by MRI $(7,8)$, whereas metabolic reductions are demonstrated in the neocortical association area by PET $(9,10)$. However, there has been controversy as to whether medial temporal metabolic reductions exist in the early stage of $\mathrm{AD}(11-16)$. The localization method using the region of interest (ROI) showed that significant medial temporal hypometabolism may be seen in $\mathrm{AD}$, even in the incipient amnestic mildcognitive-impairment stage (13-15). These morphologic and functional changes in the same patients have been studied in $\mathrm{AD}$ patients (16), although, to our knowledge, there are no reports describing these investigations in the same DLB patients.

By analyzing both MR images and glucose metabolic PET in patients with mild DLB or AD in this study—using automatic volumetric and voxel-based techniques for MR images and metabolic values of regional structures and the voxel-based technique for PET images-we delineated differences in detectable morphologic and functional abnormalities between DLB and AD. 


\section{MATERIALS AND METHODS}

All procedures followed the clinical study guidelines of our institute and were approved by the Institutional Review Board. Written informed consent was obtained from subjects and patients or patients' relatives. We studied 60 subjects, including 20 patients with very mild DLB (mean age $\pm \mathrm{SD}, 74.5 \pm 4.9$ y; 11 women, 9 men), 20 patients with very mild AD (mean age \pm SD, $74.1 \pm$ $3.3 \mathrm{y} ; 13$ women, 7 men), and 20 age-matched healthy volunteers (NC) (mean age $\pm \mathrm{SD}, 72.9 \pm 3.3 \mathrm{y} ; 15$ women, 5 men).

All patients were examined by neurologists and psychiatrists and underwent MRI of the brain, MR angiography of the neck and head, electroencephalography, and standard neuropsychologic examinations. Although the criteria for the diagnosis and management of DLB were revised in 2005 (17), we used the former criteria reported in 1996 for this study. Because the new criteria include morphologic and functional findings and this study dealt directly with morphologic and functional findings of DLB, to avoid sample selection bias, our DLB inclusion criteria were based on the DLB 1996 diagnostic criteria, which do not refer to MR images and PET findings.

The inclusion criteria for DLB were (a) a diagnosis of probable DLB using the criteria of the consortium on DLB international workshop published in 1996 (18), (b) no evidence of focal brain lesions on MR images, and (c) a mean Mini-Mental State Examination (MMSE) score of $>20$ (19).

The inclusion criteria for AD were (a) a diagnosis of probable AD using National Institute of Neurological and Communicative Disorders and Stroke/Alzheimer's Disease and Related Disorders Association criteria (20), (b) no evidence of focal brain lesions on MR images, and (c) a MMSE score of $>20$. We excluded (a) patients with complications from other neurologic diseases or those in poor overall physical condition, (b) those who had severe language, attention, or behavioral disorders that would make performance of the MRI and PET procedures difficult, and (c) those from whom informed consent could not be obtained.

The mean MMSE score \pm SD was $24.0 \pm 2.2$ in the DLB group and $24.0 \pm 2.2$ in the $\mathrm{AD}$ group. Healthy volunteers (NC), who served as control subjects, had no neurologic signs or significant medical antecedents and no abnormal findings on MR images. None of the subjects in this study had diabetes mellitus. The mean MMSE score \pm SD of the NC group was $29.8 \pm 0.6$.

\section{MRI Procedure}

The MR scanner was a 1.5-T Signa Horizon (GE Healthcare). Three-dimensional spoiled gradient echo imaging (repetition time, $14 \mathrm{~ms}$; echo time, $3 \mathrm{~ms}$; flip angle, $20^{\circ}$; thickness, $1.5 \mathrm{~mm} ; 124$ slices) was performed. MR images were obtained 0-28 d before the PET examinations.

\section{PET Procedure}

The PET procedure is described in detail elsewhere (21). In brief, ${ }^{18}$ F-FDG PET images were obtained using a Headtome IV scanner (Shimadzu Corp.). Before the PET scans, all subjects received MR examinations for PET positioning. All subjects fasted for at least $4 \mathrm{~h}$ before PET. Subjects were studied in the resting condition, with eyes closed but without ears plugs. After obtaining a transmission scan, a 12-min emission scan was started $60 \mathrm{~min}$ after intravenous injection of $185-370 \mathrm{MBq}$ of ${ }^{18} \mathrm{~F}-\mathrm{FDG}$. Data were collected in $128 \times 128$ matrices. The slice interval was 6.5 $\mathrm{mm}$ when the $z$-motion mode was used.

\section{Data Analysis}

Voxel-Based Comparison. All MR images and PET data were transferred to a personal computer. Regional brain volume was measured with a combination of software using normalizing and segmentation techniques for SPM5 ([SPM = statistical parametric mapping] Wellcome Department of Cognitive Neurology, London, United K. [http://www.fil.ion.ucl.ac.uk/spm/software/spm5/]) (2224). For PET, all individual PET images were transformed into a standard stereotactic anatomic space. Then, all images were smoothed using an isotropic gaussian kernel $(12 \mathrm{~mm}$, full width at half maximum [FWHM]) to increase the signal-to-noise ratio and to compensate for the differences in gyral anatomy among individuals. Individual ${ }^{18} \mathrm{~F}$-FDG images were adjusted using proportional scaling to a mean value of $5.0 \mathrm{mg} / 100 \mathrm{~mL} / \mathrm{min}$. For MRI, voxel-based morphometry (VBM) was used (25). After anatomic normalization, the images were automatically segmented using a cluster analysis technique. The image sets of gray matter (GM) were smoothed with an isotropic gaussian filter (12 $\mathrm{mm}, \mathrm{FWHM}$ ), and individual global counts were normalized by proportional scaling to a mean value of 5.0. These PET and MRI datasets from DLB patients, AD patients, and NC subjects were compared on a voxel-by-voxel basis using 1-way ANOVA. For direct comparison between DLB and AD, a 2-sample $t$ test was performed between GM images in DLB and AD groups and between PET images in DLB and AD groups. Significance was accepted if the voxels survived a corrected threshold of $P<0.05$ or an uncorrected threshold of $P<0.001$.

Volumetry and Relative Glucose Metabolism. Volumetry and measuring ${ }^{18} \mathrm{~F}$-FDG accumulation were performed with our modified automatic volumetric measurement of segmented brain images system (AVSIS) (26). In brief, before this analysis, voxels of interest (VOIs) templates for intracranial, hippocampi, striata, and occipital lobes were prepared. Each regional VOI template was produced on a digital phantom: the Simulated Brain Database ([SBD] http://www.bic.mni.mcgill.ca/brainweb/) in standard Montreal Neurological Institute (MNI) space, delineating the contours of each structure manually. The occipital lobe's VOI template included Brodmann areas 17, 18, and 19. The process of modified AVSIS is as follows: first, each individual MR image and PET image were coregistered, and then each subject's MR image was segmented into GM, white matter (WM), and cerebrospinal fluid (CSF) with the SPM5 segmentation program. The GM template image derived from the SBD was then spatially transformed into the individual subject's GM image, and a normalization parameter was produced. This is the same as a reversed parameter produced in anatomic normalization of individual brain to standard brain. Using this parameter, the intracranial, hippocampal, striatal, and occipital VOI templates were transformed to the individual subject's space. The total intracranial volume (TIV) area was adjusted with an image derived from the segmented GM, WM, and CSF images. The segmented images were calculated as follows: WM and GM areas were calculated with the voxels in the TIV VOI template, GM volumes in the hippocampi and striata were calculated using the transformed hippocampal and striatal VOI templates, whereas volumes of GM and WM in the occipital lobes were calculated using the transformed occipital VOI template for each subject. Each regional relative volume was calculated by normalization relative to the TIV. Next, hippocampal, striatal, and occipital ${ }^{18} \mathrm{~F}-\mathrm{FDG}$ uptake counts were calculated by determining the regions of coregistered ${ }^{18} \mathrm{~F}-\mathrm{FDG}$ images on each extracted area using the described volumetric procedure (Fig. 1). Because 
FIGURE 1. Algorithm for calculating regional structural volume and glucose metabolism An example of calculating hippocampal volume and metabolism is shown. Hippocampal VOI template is transformed to individual brain and, using this VOI template on individual segmented MR image, hippocampi are extracted and hippocampal volume is calculated. Then, using extracted hippocampi as a hippocampal VOI for individual coregistered PET image, hippocampal counts representing glucose metabolism are determined.

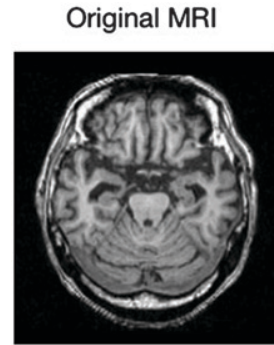

Transformed Hippocampal ROI
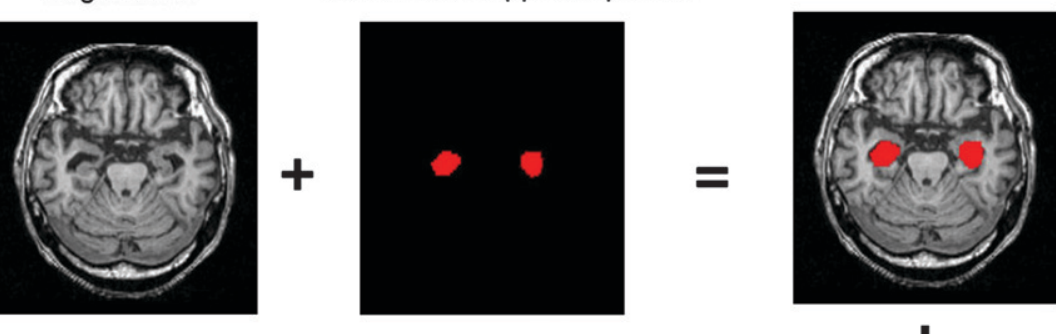

Co-registered PET image
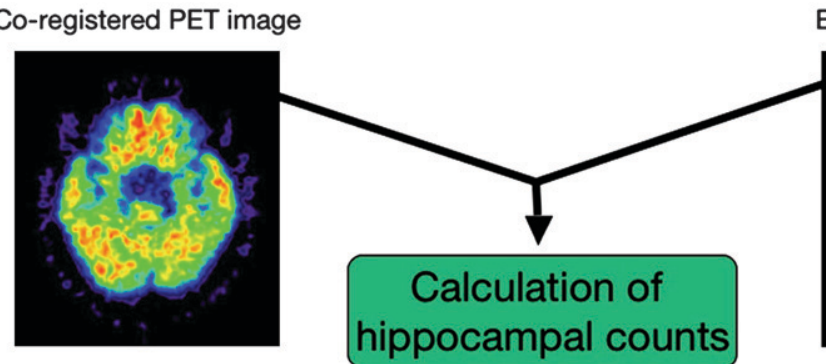

pontine and sensorimotor glucose metabolisms are relatively preserved in DLB (1), we used these regions for reference regions for calculating the relative regional cerebral glucose metabolism. Beforehand, ${ }^{18} \mathrm{~F}$-FDG PET images were transformed into the MNI space with SPM5, and then pontine and sensorimotor ${ }^{18} \mathrm{~F}-\mathrm{FDG}$ uptake counts were determined in the ROIs placed on the pons below $20-22 \mathrm{~mm}$ and the sensorimotor area over $38-42 \mathrm{~mm}$ from the anterior commissure-posterior commissure plane on the transformed MNI space. Then the extracted regional counts were normalized to the pontine counts or the sensorimotor counts and shown as relative glucose metabolism. Additionally, we calculated the affected region ratio of DLB and AD: for MRI, striatal volume/hippocampal volume (Str/Hipp), and for PET, occipital/ hippocampal counts (Occ/Hipp). Differences between groups were assessed using ANOVA followed by the post hoc Scheffé test to correct for multiple comparisons. All statistical tests were regarded as significant at $P<0.05$.

ROC Analysis for Discriminating DLB from $A D$. We also performed a receiver-operating-characteristic (ROC) analysis for discriminating DLB from $\mathrm{AD}$ with the obtained relative values for hippocampal and striatal volumes normalized to the TIV (Hipp/ TIV and Str/TIV) and hippocampal, striatal, and occipital glucose metabolisms normalized to the pontine or the sensorimotor glucose metabolism (Hipp/pons, Str/pons, and Occi/pons or Hipp/ $\mathrm{SM}, \mathrm{Str} / \mathrm{SM}$, and Occi/SM) and the affected region ratio for discriminating DLB from AD. ROC analysis was performed using ROCKIT software 0.9B (Dr. Charles E. Metz, Department of Radiology, The University of Chicago, Chicago, IL). The areas under the curves and accuracy were calculated.

\section{RESULTS}

\section{Voxel-Based Comparison}

In the MRI VBM study, the gray matter densities of the left parahippocampal gyrus and the hippocampal complex in the AD group were significantly lower than those in the NC group $(P<0.05$, corrected $)$, whereas the gray matter densities of the left putamen and caudate head in the DLB group were significantly lower than those in the NC group ( $P<0.05$, corrected) (Table 1; Fig. 2A).

PET SPM analysis demonstrated that a metabolic reduction was present in the bilateral posterior cingulate gyri in the AD group compared with that of the NC group $(P<$ 0.05 , corrected). Furthermore, in the DLB group, occipital, temporal, parietal, and frontal metabolisms were significantly reduced compared with those in the NC group $(P<$ 0.05 , corrected) (Table 1; Fig. 2A).

In direct comparison between the DLB and AD groups, VBM demonstrated a reduced left caudate volume in the DLB group compared with that in the AD group but did not show significantly reduced regions in the AD group compared with those in the DLB group (Table 1; Fig. 2B) $(P<$ 0.001 , uncorrected). SPM analysis for PET showed significantly reduced metabolism in the bilateral frontal, parietotemporal association cortices, left posterior cingulate gyrus, and bilateral occipital lobes in the DLB group compared with that in the AD group but did not show significantly reduced regions in the $\mathrm{AD}$ group compared with that in the DLB group (Table 1; Fig. 2B) $(P<0.001$, uncorrected).

\section{Volumetry and Relative Glucose Metabolism}

Mean regional volumes in each group are presented in Table 2. TIV, whole brain (WB), and hippocampal volumes in the AD group were significantly smaller than those in the NC group. The AD group's WB volume normalized to TIV did not differ significantly from that of the NC group, but the AD group's hippocampal volume normalized to TIV was significantly smaller than that in the NC group. There were no significant differences in occipital volumes among the DLB, AD, and NC groups for either the absolute or the 
TABLE 1

Peak Location of Significant GM Density Decrease of Metabolic Decrease in DLB Group and AD Group Compared with NC Group or Those in DLB Group Compared with AD Group

\begin{tabular}{|c|c|c|c|c|c|}
\hline Comparison & Location & $t$ value & $x$ & $y$ & $z$ \\
\hline \multirow[t]{2}{*}{ MRI VBM NC > DLB } & L putamen & 6.16 & -31 & 8 & 7 \\
\hline & L caudate head & 6.15 & -8 & 11 & -5 \\
\hline \multirow[t]{2}{*}{ MRI VBM NC > AD } & L parahippocampal gyrus & 5.91 & -33 & -21 & -22 \\
\hline & L hippocampus & 5.10 & -26 & -7 & -18 \\
\hline \multirow[t]{10}{*}{${ }^{18} \mathrm{~F}-\mathrm{FDG}$ PET NC $>$ DLB } & $\mathrm{R}$ posterior cingulate gyrus & 7.01 & 13 & -54 & 29 \\
\hline & $\mathrm{L}$ posterior cingulate gyrus & 4.84 & -10 & -46 & 26 \\
\hline & $\mathrm{R}$ middle temporal gyrus & 5.28 & 54 & -42 & -8 \\
\hline & $\mathrm{L}$ inferior temporal gyrus & 6.83 & -45 & -42 & -22 \\
\hline & $\mathrm{R}$ middle frontal gyrus & 6.55 & 41 & 28 & 28 \\
\hline & $\mathrm{L}$ inferior frontal gyrus & 5.92 & -47 & 21 & 18 \\
\hline & $\mathrm{R}$ inferior occipital gyrus & 5.02 & 31 & -89 & -10 \\
\hline & L lingual gyrus & 5.03 & -20 & -100 & -5 \\
\hline & $\mathrm{R}$ inferior parietal lobule & 5.31 & 45 & -58 & 34 \\
\hline & $\mathrm{L}$ inferior parietal lobule & 6.25 & -22 & 21 & 31 \\
\hline \multirow[t]{2}{*}{${ }^{18} \mathrm{~F}-\mathrm{FDG}$ PET NC $>\mathrm{AD}$} & $\mathrm{R}$ posterior cingulate gyrus & 5.73 & 1 & -38 & 26 \\
\hline & $\mathrm{L}$ posterior cingulate gyrus & 4.94 & -8 & -44 & 24 \\
\hline MRI VBM AD > DLB & L caudate head & 4.46 & -13 & 12 & 0 \\
\hline \multirow[t]{7}{*}{${ }^{18} \mathrm{~F}-\mathrm{FDG}$ PET AD > DLB } & L lingual gyrus & 4.80 & -17 & -89 & -10 \\
\hline & $\mathrm{R}$ lingual gyrus & 3.96 & 15 & -79 & -8 \\
\hline & $L$ inferior frontal gyrus & 4.20 & -47 & 16 & 29 \\
\hline & $\mathrm{R}$ precuneus & 4.54 & 18 & -54 & 32 \\
\hline & $\mathrm{L}$ middle temporal gyrus & 4.27 & -41 & -36 & -7 \\
\hline & $\mathrm{R}$ middle frontal gyrus & 4.05 & 47 & 30 & 29 \\
\hline & $\mathrm{L}$ middle frontal gyrus & 4.61 & -43 & 22 & 33 \\
\hline
\end{tabular}

$x=$ distance $(\mathrm{mm})$ to right $(+)$ or left $(-)$ of midsagittal line; $y=$ distance anterior $(+)$ or posterior $(-)$ to vertical plane through anterior commissure; $z$ = distance above $(+)$ or below $(-)$ intercommissural line.

Coordinates are in millimeters, relative to anterior commissure, corresponding to atlas of Talairach and Tournoux (27).

relative values normalized to TIV. Absolute and relative striatal volumes (Str/TIV) in the DLB group were significantly smaller than those in the NC group, and striatal volumes (Str/TIV) in the DLB group were significantly smaller than those in the $\mathrm{AD}$ group.

Occipital glucose metabolism normalized to that of the pons or sensorimotor area was significantly reduced in the DLB compared with that of the AD and NC groups. Hippocampal metabolism normalized to that of the pons or sensorimotor area was also significantly reduced in the $\mathrm{AD}$ group compared with that of the DLB and NC groups (Table 2).

The affected region ratios of MRI and PET are shown in Table 3. The MRI Str/Hipp ratio in the AD group was significantly higher than that of the DLB and NC groups, and the PET Occ/Hipp ratio in the DLB group was significantly lower than that of the $\mathrm{AD}$ and $\mathrm{NC}$ groups.

\section{ROC Analysis for Discriminating DLB from AD}

Accuracies and areas under the ROC curve for discriminating DLB from AD by each value are shown in Table 4. The areas under the ROC curve and the accuracy for PET Occ/Hipp were the highest among those of other values, though there were no significant differences in the partial area index for ROC curves among these parameters. Figure 3 shows the ROC curves of the MRI Hipp/TIV, Str/TIV, PET Hipp/pons, and Occ/pons and the affected regional ratio of MRI Str/Hipp and PET Occ/Hipp for discriminating DLB from AD.

\section{DISCUSSION}

To date, ${ }^{18} \mathrm{~F}-\mathrm{FDG}$ PET and MR images obtained in mild DLB and NC subjects have been studied separately, not in the same individuals. To our knowledge, our study is the first in which ${ }^{18} \mathrm{~F}$-FDG PET and MR images from the same individuals with mild DLB were studied and compared with those of individual AD subjects. We were able to show that the areas of reduced metabolism and diminished GM volume are not the same in these 2 neurodegenerative diseases.

\section{Voxel-Based Analysis}

VBM analysis showed significant striate atrophy in DLB and hippocampal atrophy in $\mathrm{AD}$, even in the mild stages of these diseases, though only on the left side. SPM analysis of ${ }^{18}$ F-FDG PET findings revealed significant glucose metabolic reductions in the occipital, temporoparietal, and frontal association cortices in the DLB. Thus, even in the mild stage of DLB, glucose metabolic disturbances are widespread compared with those of healthy aged subjects. 
A MRI VBM

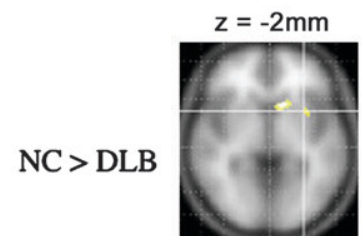

$z=-24 m m$

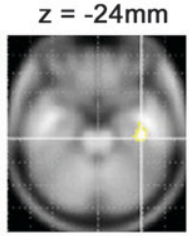

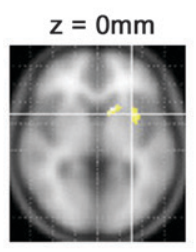

$z=-22 m m$

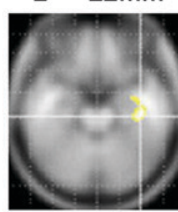

MRI VBM

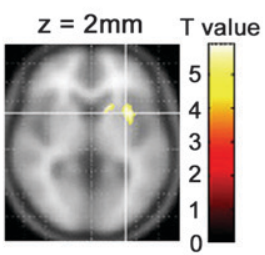

$z=-20 m m \quad T$ value

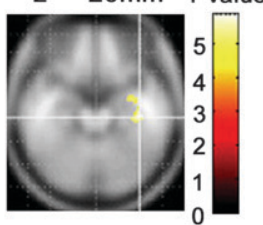

$\mathrm{NC}>\mathrm{DLB}$

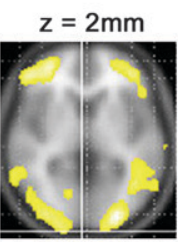

$z=24 \mathrm{~mm}$

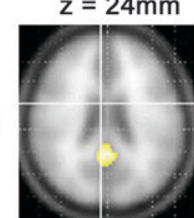

${ }^{18}$ F-FDG PET

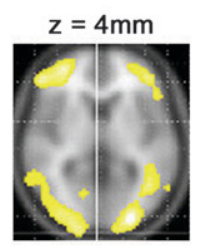

$z=26 \mathrm{~mm}$

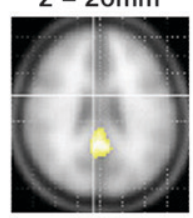

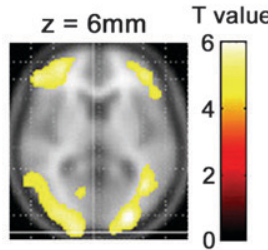

$\mathrm{z}=28 \mathrm{~mm} \quad T$ value

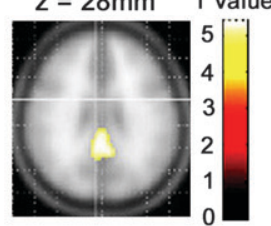

B
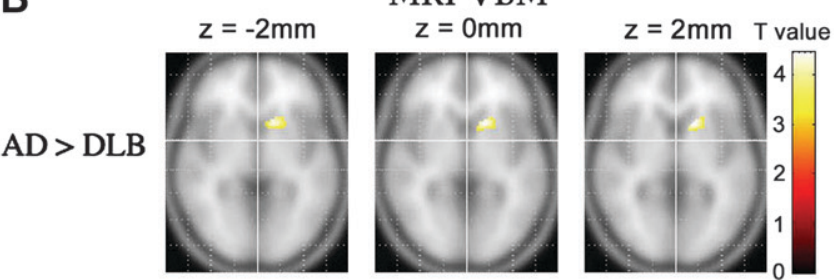

${ }^{18}$ F-FDG PET

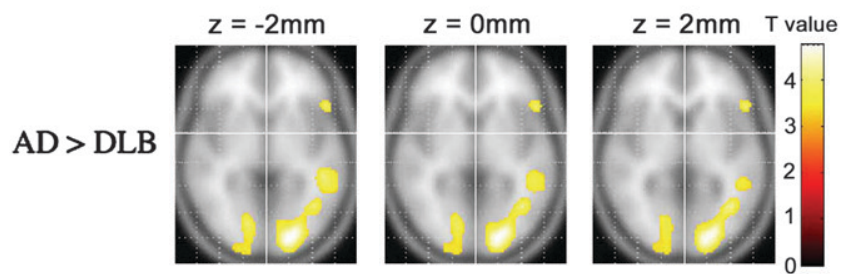

FIGURE 2. Voxels with significantly lower GM density or glucose metabolism are shown in axial plane of standard brain. (A) Left caudate and putamen GM densities in DLB group were significantly lower than those of NC group (upper left row). Left hippocampal and parahippocampal GM densities in AD group were significantly lower than those of NC group (lower left row). Bilateral occipital, temporoparietal, and frontal glucose metabolisms in DLB group were significantly lower than those in NC group (upper right row), and parietal and posterior cingulated metabolisms were also decreased (not shown). Bilateral posterior cingulate glucose metabolism in AD group was significantly lower than that in NC group (lower right row) $(P<0.05$, corrected). (B) Left caudate GM densities in DLB group were significantly lower than those of AD group (upper row). Bilateral occipital glucose metabolism was significantly decreased in DLB compared with that in AD (lower row), and glucose metabolism in bilateral frontal, parietotemporal association cortices and left posterior cingulate gyrus, was also significantly reduced in DLB (not shown) $(P<$ 0.001 , uncorrected).

SPM analysis of mild AD brains also demonstrated posterior cingulate glucose metabolic reduction, an observation that supports previous reports $(10,15,16)$. Our study demonstrated in the DLB brain not only previously identified posterior hypometabolism, including the posterior cingulate and occipital regions, but also frontal hypometabolism. The metabolic reduction pattern of DLB is relatively similar to that of $\mathrm{AD}$, with association of occipital metabolic reduction; however, the degree of metabolic reduction in those areas is larger than that of $\mathrm{AD}$, which has been reported in moderate DLB (1). Our study showed the same pathophysiology even in mild DLB. We emphasized that mild DLB shows abnormalities involving vast portions of the entire cortex compared with mild AD. Thus, it appears that the process of neurodegeneration may be more advanced in the mild DLB group compared with the mild AD group, in spite of comparable neuropsychologic results. There are 2 different aspects: (a) better cognitive reserve in the DLB group or (b) insufficient sensitivity of the applied neuropsychologic testing-MMSE to detect the true extent of neurodegeneration in DLB. In any case, it cannot be excluded that the observed findings may in part be due to different stages of disease in using MMSE scores.

\section{Medial Temporal Lobe}

The medial temporal area is histopathologically affected in the early stages of AD. This medial temporal change has been described in previous reports using MRI $(7,8)$ and was confirmed in the present study of patients with very mild AD. Morphologic imaging by MRI clearly demonstrates macropathologic changes in mild AD. However, previous PET studies have shown that medial temporal glucose 
TABLE 2

Mean Volumes and Glucose Metabolism of Each Region

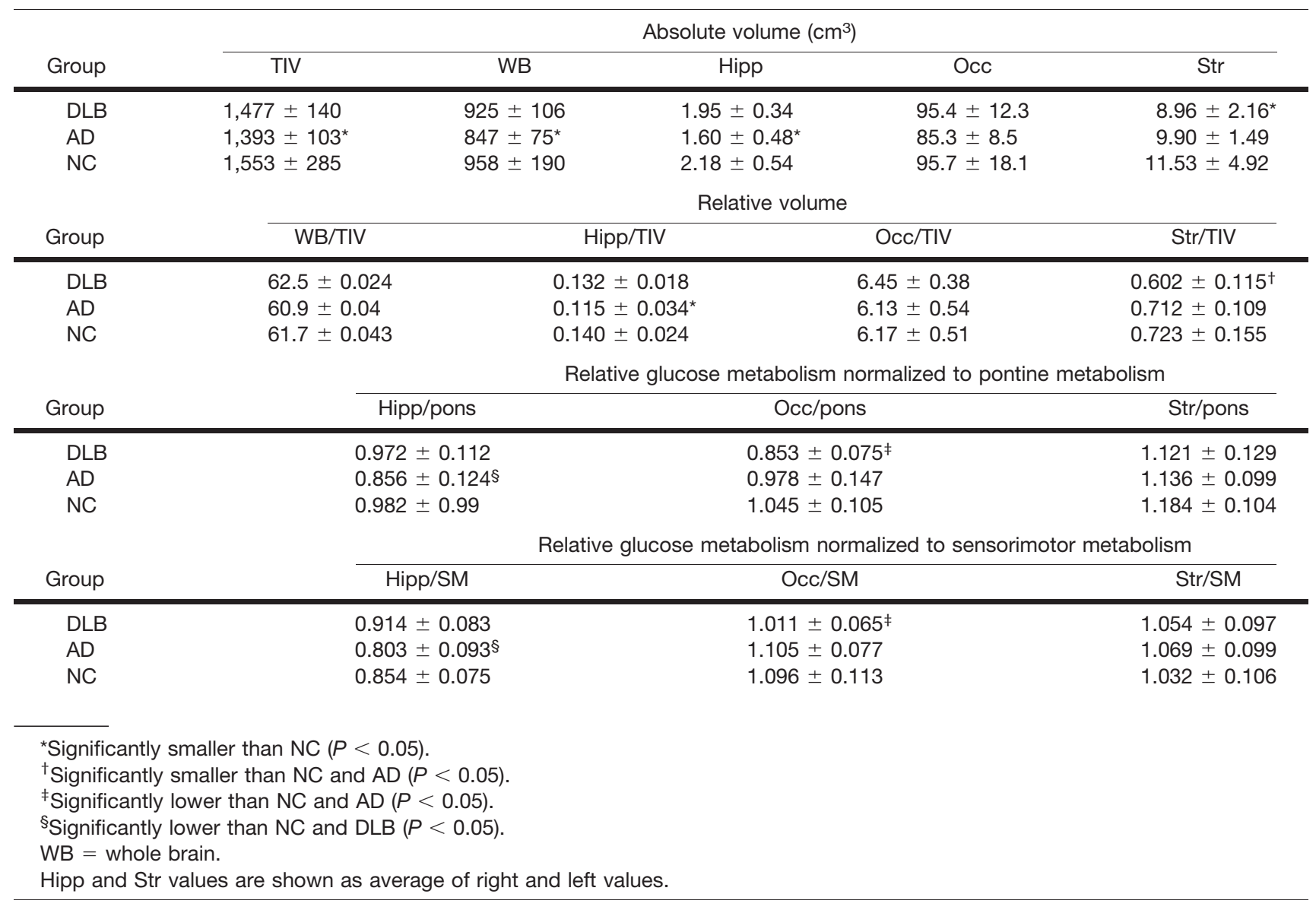

metabolism and blood flow are preserved in mild-tomoderate AD patients $(11,28)$. Recently, Nestor et al. in 2003 (14) and Mosconi et al. in 2005 (15) suggested that hippocampal metabolism in patients with mild cognitive impairment and $\mathrm{AD}$ was reduced, based on results obtained using their technique of drawing ROIs on MR images, and described the voxel-based analysis using SPM as failing to show the hippocampal metabolic reduction. Herein, we verified their findings using our new VOI warping method combined with MRI. In mild AD patients, SPM did not demonstrate hippocampal metabolic reduction, whereas our method showed significantly reduced hippocampal metabolism. However, in mild DLB, neither SPM nor our method

TABLE 3 Affected Region Ratios

\begin{tabular}{lll}
\hline Group & MRI: Str/Hipp & PET: Occ/Hipp \\
\hline DLB & $4.589 \pm 0.792$ & $0.884 \pm 0.085^{\star}$ \\
AD & $6.707 \pm 2.051^{\dagger}$ & $1.151 \pm 0.154$ \\
NC & $5.310 \pm 1.556$ & $1.096 \pm 0.108$
\end{tabular}

*Significantly lower than NC and AD $(P<0.01)$.

${ }^{\dagger}$ Significantly higher than NC and DLB $(P<0.01)$. demonstrated this reduction in hippocampal metabolism. As to structural change, no hippocampal volume reduction was demonstrated in DLB using either SPM or our method. Therefore, in mild AD, but not mild DLB, both metabolic and structural alterations are present. These findings support previous results obtained by studying functional and morphologic changes separately. There was a 5\% reduction in the Hipp/SM ratio in $\mathrm{AD}$ compared with $\mathrm{NC}$ but there was a $6 \%$ increase in this ratio when DLB was compared with NC (Table 1). It seems highly unlikely that DLB is associated with hippocampal hypermetabolism. This suggests that the increase in the ratio is actually due to a decrease in the denominator-that is, there is a degree of SM

TABLE 4

Area Under ROC Curves and Accuracy for Discriminating DLB from AD by Each Value

\begin{tabular}{llcc}
\hline Method & Ratio & $\mathrm{A}_{z}$ & Accuracy (\%) \\
\hline MRI & Hipp/TIV & 0.694 & 62 \\
& Str/TIV & 0.763 & 71 \\
PET & Str/Hipp & 0.864 & 80 \\
& Hipp/pons & 0.708 & 66 \\
& Occ/pons & 0.790 & 73 \\
& Occ/Hipp & 0.941 & 87 \\
\hline
\end{tabular}



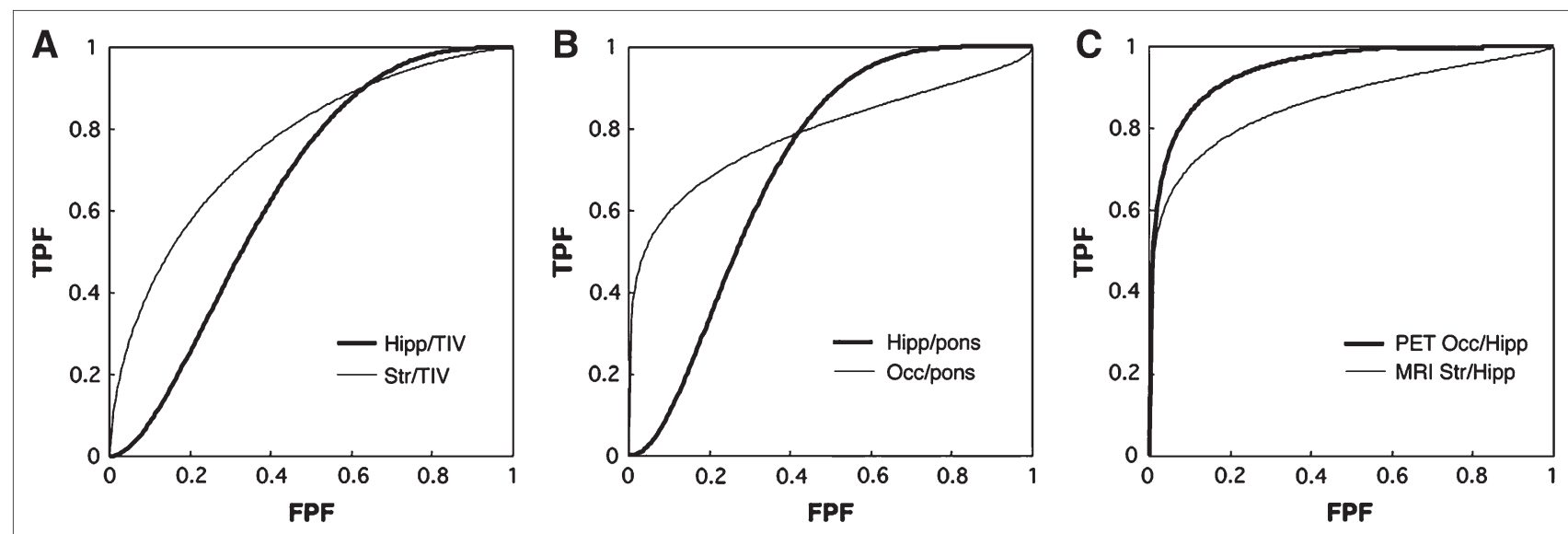

FIGURE 3. ROC curves for PET and MRI diagnostic performances in discriminating DLB from AD. Area under ROC curve of Occ/ Hipp by PET (area under the curve $\left[A_{z}\right]=0.941$ ) was the largest among all values. $(A)$ Relative regional volumes of hippocampi (Hipp/TIV) and striata (Str/TIV) by MRI. (B) Relative regional glucose metabolism in hippocampi (Hipp/pons) and occipital lobes (Occ/pons) by ${ }^{18} \mathrm{~F}-\mathrm{FDG}$ PET. (C) Affected regional ratio obtained by PET (Occ/Hipp) and MRI (Str/Hipp). TPF = true-positive fraction $=$ sensitivity; FPF $=$ false-positive fraction $=1-$ specificity.

hypometabolism in DLB consequently due to a widespread metabolic reduction even in the mild DLB brain. Therefore, as the Hipp/pons ratio showed, hippocampal metabolism in mild DLB seems not increased but relatively preserved.

\section{Striatum}

In the striatum, dopamine transporter imaging is reportedly useful for distinguishing between DLB and AD because, in the former, dopamine transporter uptake in the striatum is low $(29,30)$. Though low dopamine transporter uptake has been shown in the DLB striatum, to our knowledge, there are no reports indicating that striatal metabolism or perfusion is altered. Glucose metabolism in the striatum was reported to be preserved in DLB, based on measuring absolute glucose metabolism (1). Our study verified that relative striatal metabolism was also preserved in the mild DLB brain. We showed reduced striatal volume in DLB brains compared with those affected by AD and in NC subjects. Barber et al. reported that volumetric MRI analysis of the caudate nucleus does not discriminate among patients with DLB, AD, and vascular dementia (31). Almeida et al. also found that caudate atrophy is not region specific but, rather, is part of generalized brain volume loss (32). On the contrary, Cousins et al. showed that patients with DLB had significantly smaller ratios of putamen volume to TIV than both control subjects and patients with $\mathrm{AD}$ and, furthermore, that the $\mathrm{AD}$ patients did not differ from control subjects in any measure of the putamen volume (33). Our study supports their findings indicating that striatal volume in the DLB brain is significantly smaller than those in $\mathrm{AD}$ and $\mathrm{NC}$ brains. It is interesting that, in spite of volume loss of striatum, there is no glucose metabolic reduction in DLB. The pathophysiology of this situation is unclear. This may be due to upregulation of the low dopamine uptake.

\section{Occipital Lobe}

In the DLB brain, occipital glucose metabolism is affected despite the absence of morphologic change, though the metabolic and morphologic features have been studied separately. Gross structural changes in the occipital lobe reportedly do not occur in patients with mild-to-moderate DLB or AD, based on results obtained using MRI volumetric techniques (34). Our study demonstrates these findings in individual subjects. On the contrary, using VBM, Burton et al. reported that occipital atrophy in Parkinson disease dementia (PDD) was the only difference between patients with Parkinson's disease and a PDD group (35). Although PDD is thought to be in the same spectrum as DLB, no previous reports have demonstrated significant occipital atrophy in DLB. Our VBM study and volumetric study confirmed the latter findings. Burton et al. also noted that uncertainty remains with regard to the relationships between atrophy, neuropathologic changes, and perfusion deficits in Parkinson's disease with and without dementia (35). Our study confirmed morphologic and functional discrepancies in the same individual DLB brains.

\section{Differential Diagnosis for AD and DLB}

For routine clinical examination, conventional ROI placement or a voxel-based technique, identifying reduced occipital glucose metabolism, may be worthwhile because, as shown in the present study, these techniques are moderately useful for distinguishing mild DLB from mild AD. Moreover, our ROC analysis demonstrated that the PET Occ/Hipp value - which was amplified with combination of each DLB's and AD's affected value obtained with ${ }^{18} \mathrm{~F}-$ FDG PET - has higher accuracy than other values. Herein, we used software combining ${ }^{18} \mathrm{~F}-\mathrm{FDG}$ PET and MRI with extraction of regional structures such as the hippocampus. 


\section{CONCLUSION}

We demonstrated that morphologically and metabolically affected regions differ between very mild stages of DLB and AD. Our findings suggest that a combined morphologic and functional approach using both MRI and PET may improve the accuracy of early diagnosis of very mild DLB and AD. We anticipate further study in a clinical setting to confirm the potential of combining these 2 imaging modalities.

\section{REFERENCES}

1. Ishii K, Imamura T, Sasaki M, et al. Regional cerebral glucose metabolism in dementia with Lewy bodies and Alzheimer's disease. Neurology. 1998;51:125130 .

2. Lobotesis K, Fenwick JD, Phipps A, et al. Occipital hypoperfusion on SPECT in dementia with Lewy bodies but not AD. Neurology. 2001;56:643-649.

3. Ishii K, Hosaka K, Mori T, Mori E. Comparison of FDG-PET and IMP-SPECT in patients with dementia with Lewy bodies. Ann Nucl Med. 2004;18:447-451.

4. Kosaka K, Yoshimura M, Ikeda K, Budka H. Diffuse type of Lewy body disease: progressive dementia with abundant cortical Lewy bodies and senile changes of varying degree-A new disease? Clin Neuropathol. 1984;3:185-192.

5. Perry RH, Irving D, Blessed G, Fairbairn A, Perry EK. Senile dementia of Lewy body type: a clinically and neuropathologically distinct form of Lewy body dementia in the elderly. J Neurol Sci. 1990;95:119-139.

6. Mega MS, Masterman DL, Benson DF, et al. Dementia with Lewy bodies: reliability and validity of clinical and pathologic criteria. Neurology. 1996;47: 1403-1409.

7. Jack CR, Petersen RC, O'Brien PC, Tangalos EG. MR-based hippocampal volumetry in the diagnosis of Alzheimer's disease. Neurology. 1992;42:183-188.

8. Pennanen C, Kivipelto M, Tuomainen S, et al. Hippocampus and entorhinal cortex in mild cognitive impairment and early AD. Neurobiol Aging. 2004;25: 303-310.

9. Herholz K, Perani D, Salmon E, et al. Comparability of FDG PET studies in probable Alzheimer's disease. J Nucl Med. 1993;34:1460-1466.

10. Hoffman JM, Welsh-Bohmer KA, Hanson M, et al. FDG PET imaging in patients with pathologically verified dementia. J Nucl Med. 2000;41:1920-1928.

11. Ishii K, Sasaki M, Yamaji S, Sakamoto S, Kitagaki H, Mori E. Relatively preserved hippocampal glucose metabolism in mild Alzheimer's disease. Dement Geriatr Cogn Disord. 1998;9:317-322.

12. Minoshima S, Giordani B, Berent S, Frey KA, Foster NL, Kuhl DE. Metabolic reduction in the posterior cingulate cortex in very early Alzheimer's disease. Ann Neurol. 1997;42:85-94.

13. De Santi S, de Leon MJ, Rusinek H, et al. Hippocampal formation glucose metabolism and volume losses in MCI and AD. Neurobiol Aging. 2001;22: 529-539.

14. Nestor PJ, Fryer TD, Smielewski P, Hodges JR. Limbic hypometabolism in Alzheimer's disease and mild cognitive impairment. Ann Neurol. 2003;54: 343-351.

15. Mosconi L, Tsui WH, De Santi S, et al. Reduced hippocampal metabolism in MCI and AD: automated FDG-PET image analysis. Neurology. 2005;64:18601867.

16. Ishii K, Sasaki H, Kono AK, Miyamoto N, Fukuda T, Mori E. Comparison of gray matter and metabolic reduction in mild Alzheimer's disease using FDG-
PET and voxel-based morphometric MR studies. Eur J Nucl Med Mol Imaging. 2005;32:959-963.

17. McKeith IG, Dickson DW, Lowe J, et al. Diagnosis and management of dementia with Lewy bodies: third report of the DLB Consortium. Neurology. 2005;65:1863-1872.

18. McKeith IG, Galasko D, Kosaka K, et al. Consensus guidelines for the clinical and pathologic diagnosis of dementia with Lewy bodies (DLB): report of the consortium on DLB international workshop. Neurology. 1996;47:1113-1124.

19. Folstein MF, Folstein SE, McHugh PR. "Mini-mental state": a practical method for grading the cognitive state of patients for the clinician. J Psychiatr Res. 1975;12:189-198.

20. McKhann G, Drachman D, Folstein M, Katzman R, Price D, Stadlan EM. Clinical diagnosis of Alzheimer's disease: report of the NINCDS-ADRDA Work Group under the auspices of Department of Health and Human Services Task Force on Alzheimer's disease. Neurology. 1984;34:939-944.

21. Ishii K, Sasaki M, Kitagaki H, et al. Reduction of cerebellar glucose metabolism in advanced Alzheimer's disease. J Nucl Med. 1997;38:925-928.

22. Friston KJ, Ashburner J, Frith CD, Poline J-B, Heather JD, Frackowiak RSJ. Spatial registration and normalization of images. Hum Brain Mapp. 1995;2: $165-189$.

23. Ashburner J, Neelin P, Collins DL, Evans AC, Friston KJ. Incorporating prior knowledge into image registration. Neuroimage. 1997;6:344-352.

24. Ashburner J, Friston KJ. Nonlinear spatial normalization using basis functions. Hum Brain Mapp. 1999;7:254-266.

25. Ashburner J, Friston KJ. Voxel-based morphometry: the methods. Neuroimage. 2000;11:805-821.

26. Ishii K, Soma T, Kono AK, et al. Automatic volumetric measurement of segmented brain structures on magnetic resonance imaging. Radiat Med. 2006; 24:422-430.

27. Talairach L, Tournoux P. Co-Planar Sterectaxic Atlas of the Human Brain: 3-D Proportional System-An Approach to Cerebral Imaging. Stuttgart, Germany: Thieme Verlag; 1988.

28. Ishii K, Sasaki M, Yamaji S, Sakamoto S, Kitagaki H, Mori E. Paradoxical hippocampus perfusion in mild-to-moderate Alzheimer's disease. J Nucl Med. 1998;39:293-298.

29. O'Brien JT, Colloby S, Fenwick J, et al. Dopamine transporter loss visualized with FP-CIT SPECT in the differential diagnosis of dementia with Lewy bodies. Arch Neurol. 2004;61:919-925.

30. Walker Z, Costa DC, Walker RW, et al. Differentiation of dementia with Lewy bodies from Alzheimer's disease using a dopaminergic presynaptic ligand. J Neurol Neurosurg Psychiatry. 2002;73:134-140.

31. Barber R, McKeith I, Ballard C, O'Brien J. Volumetric MRI study of the caudate nucleus in patients with dementia with Lewy bodies, Alzheimer's disease, and vascular dementia. J Neurol Neurosurg Psychiatry. 2002;72:406-407.

32. Almeida OP, Burton EJ, McKeith I, Gholkar A, Burn D, O’Brien JT. MRI study of caudate nucleus volume in Parkinson's disease with and without dementia with Lewy bodies and Alzheimer's disease. Dement Geriatr Cogn Disord. 2003;16: 57-63.

33. Cousins DA, Burton EJ, Burn D, Gholkar A, McKeith IG, O'Brien JT. Atrophy of the putamen in dementia with Lewy bodies but not Alzheimer's disease: an MRI study. Neurology. 2003;61:1191-1195.

34. Middelkoop HA, van der Flier WM, Burton EJ, et al. Dementia with Lewy bodies and AD are not associated with occipital lobe atrophy on MRI. Neurology. 2001; 57:2117-2120.

35. Burton EJ, McKeith IG, Burn DJ, Williams ED, O'Brien JT. Cerebral atrophy in Parkinson's disease with and without dementia: a comparison with Alzheimer's disease, dementia with Lewy bodies and controls. Brain. 2004;127:791-800. 